Proceedings

\title{
Humidity Sensitivity and Coil Design of a High-Precision Eddy-Current Displacement Sensor ${ }^{\dagger}$
}

\author{
Johan G. Vogel *, Vikram Chaturvedi and Stoyan Nihtianov \\ Electronic Instrumentation Laboratory, Delft University of Technology, Mekelweg 4, 2628CD Delft, \\ The Netherlands; v.chaturvedi@tudelft.nl (V.C.); s.nihtianov@tudelft.nl (S.N.) \\ * Correspondence: j.g.vogel@tudelft.nl; Tel.: +31-(0)15-278-9177 \\ + Presented at the Eurosensors 2017 Conference, Paris, France, 3-6 September 2017.
}

Published: 28 August 2017

\begin{abstract}
Unlike capacitive displacement sensors, Eddy-Current Displacement Sensors (ECDSs) possess an inherently low sensitivity to environmental conditions, such as the humidity of the ambient air. By elevating the excitation frequency it is possible to mitigate their major limitations regarding stability and resolution, making them of interest for high-precision displacement sensing. However, by increasing the excitation frequency, ECDSs become less immune to environmental conditions, due to the inevitable parasitic capacitance of the sensing coil. In this work, we formulate a requirement for the minimum Self-Resonance Frequency (SRF) of the coil, based on the specified humidity variation and the allowable displacement error. This requirement provides an input for the design of the high-precision ECDS probe.
\end{abstract}

Keywords: displacement sensor; eddy-current sensor; parasitic capacitance; self-resonance frequency; humidity sensitivity; planar coil

\section{Introduction}

In many application fields, such as aerospace and lithography, high-precision displacement sensors are utilised. If a high resolution over only a short displacement range is required, often capacitive sensors are selected. An important limitation of capacitive sensors, however, is their sensitivity to environmental conditions, such as contamination and humidity of the ambient air [1].

As an alternative to capacitive sensors, Eddy-Current Displacement Sensors (ECDSs) can be utilised, but state-of-the-art industrial sensors typically have a relatively poor stability and a low resolution, due to their relatively low excitation frequency of up to several megahertz. These limitations can be mitigated by using a much higher excitation frequency, i.e., above $100 \mathrm{MHz}[2,3]$. In our earlier work, an ECDS was developed that uses an excitation frequency of $126 \mathrm{MHz}$. The sensor achieves a resolution of $0.6 \mathrm{~nm}$ at $2 \mathrm{kHz}$ signal bandwidth [4].

The developed ECDS uses the inductance of the sensing coil as a measure of standoff distance from the conductive target. Apart from inductance, the coil also exhibits parasitic interwinding capacitance (Figure 1a), leading to a certain Self-Resonance Frequency (SRF) [5]. Unfortunately, this capacitance is sensitive to changes in the relative permittivity [6]. This paper studies how humidity change influences the displacement measurement and shows how the required coil SRF relates to specifications regarding allowable humidity variation and displacement measurement error.

In Section 2, the sensor's sensitivity to parasitic capacitance change is analysed using circuit theory. Section 3 studies the sensitivity to humidity change using a combination of analytic equations and finite element modelling. 


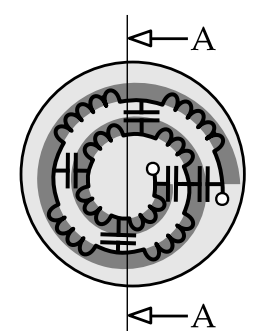

probe front

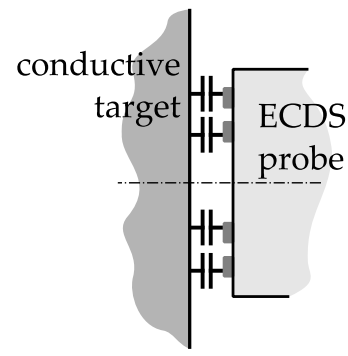

cross-section $A-A$

(a)

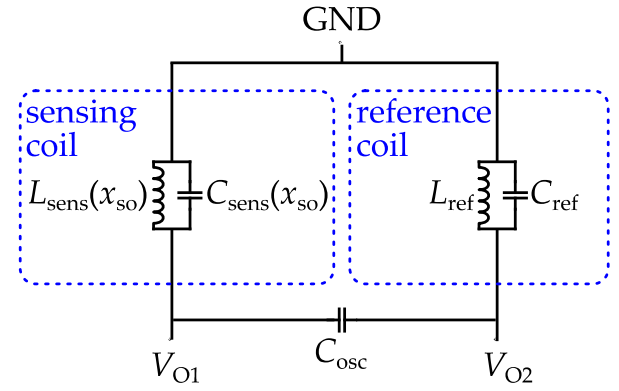

(b)

Figure 1. (a) Front view and cross-section of an ECDS probe with a flat sensing coil. The coil not only has an inductance that varies with standoff $x_{\mathrm{so}}$, but also has a parasitic interwinding capacitance;

(b) Schematic of the sensor's front-end [4].

\section{Sensitivity to Parasitic Capacitance Change}

Figure $1 \mathrm{~b}$ shows the circuit of the sensor's self-oscillating frontend reported in [4]. It consist of a sensing coil, a reference coil and an on-chip capacitor $\left(C_{\text {osc }}\right)$ that together form an oscillator. The inductance of the sensing coil is a measure for the standoff $x_{\mathrm{so}}$ from a conductive measurement target. The reference coil is placed at a fixed standoff from a reference target.

The electronic readout measures the reactance of each coil, which not only depends on the inductance, but also on the lumped parasitic capacitances of the coil. The inductance and parasitic capacitance of the sensing coil are both a function of the standoff; frequency dependency is neglected.

The sensing coil has an impedance of

$$
Z_{\text {sens }}=\frac{j \omega_{\text {exc }} L_{\text {sens }}}{-\omega_{\text {exc }}^{2} L_{\text {sens }} C_{\text {sens }}+1}
$$

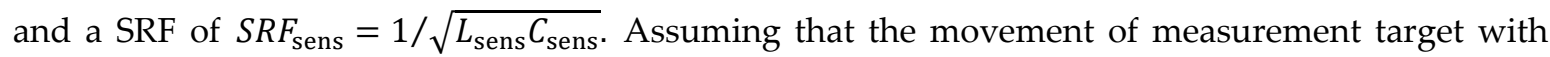
respect the sensing coil is relatively small compared to the nominal standoff, their inductance and their parasitic capacitance can be assumed to be almost equal, so that $L_{\text {meas }} \cong L_{\text {ref }}=L$ and $C_{\text {meas }} \cong C_{\text {ref }}=C$. The total impedance of the coils is then

$$
Z=\frac{2 j \omega L}{-\omega^{2} 2 L\left(C_{\text {osc }}+1 / 2 C\right)+1},
$$

leading to an excitation frequency of $\omega_{\mathrm{exc}}=1 / \sqrt{2 L\left(C_{\mathrm{osc}}+1 / 2 C\right)}$.

If the humidity of the environment changes, the capacitance of the sensing coil changes, but the capacitance of the reference coil remains constant. Thus, the capacitance change of the sensing coil directly leads to a measurement error. Neglecting the relatively small change of the excitation frequency, the sensitivity of the impedance to the sensing coil's parasitic capacitance becomes

$$
\frac{\partial Z_{\text {sens }}}{\partial C_{\text {sens }}}=\frac{j \omega_{\text {exc }}^{3} L_{\text {sens }}^{2}}{\left(1-\omega_{\text {exc }}^{2} L_{\text {sens }} C_{\text {sens }}\right)^{2}}=\frac{j \omega_{\text {exc }}^{3} L_{\text {sens }}^{2}}{\left(1-\alpha^{-2}\right)^{2}}
$$

where $\alpha=S R F / \omega_{\text {exc }}$ denotes the SRF normalised by the excitation frequency (both in $\mathrm{rad} / \mathrm{s}$ ). The resulting displacement error can be derived by converting Equation (3) to equivalent inductance and dividing it by the displacement sensitivity $\left(S_{x}=\partial L_{\text {sens }} / \partial x\right)$ :

$$
\frac{\partial x_{\mathrm{so}}}{\partial C_{\mathrm{sens}}}=\frac{1}{\omega_{\mathrm{exc}} S_{x}} \frac{\partial X_{\mathrm{sens}}}{\partial C_{\mathrm{sens}}}=\frac{\omega_{\mathrm{exc}}^{2} L_{\mathrm{sens}}^{2}}{\left(1-\alpha^{-2}\right)^{2} S_{x}} .
$$

\section{Sensitivity to Humidity Variation}

To obtain the sensitivity of the displacement measurement to humidity variation, the sensitivity $\partial C_{\text {sens }} / \partial H$ need to be found. $\partial C_{\text {sens }} / \partial H$ can be decomposed into the sensitivities $\partial C / \partial \varepsilon_{\mathrm{r}}$ and $\partial \varepsilon_{\mathrm{r}} / \partial H$, with $\varepsilon_{\mathrm{r}}$ the relative permittivity of the air volume, which changes with humidity, and $H$ the relative humidity in percent. 
Lea et al. derived the following relation for the relative permittivity of humid air [7]:

$$
\varepsilon_{\mathrm{r}}(H)=\frac{a_{1}}{T\left(P+\frac{a_{2} P_{s}}{T} H\right) 10^{-6}},
$$

with $a_{1}=1.58 \mathrm{~K} / \mathrm{Pa}, a_{2}=48 \mathrm{~K}, T$ the temperature in Kelvin and $P_{s}$ the vapour pressure of water, which can be calculated using the August-Roche-Magnus formula [8]:

$$
P_{S}[\mathrm{~Pa}]=610.94 \exp \left(17.625 \frac{T-273.15}{T-30.11}\right) .
$$

Thus, at room temperature $(T=293.15 \mathrm{~K})$, the sensitivity of the relative permittivity to the relative humidity is $\partial \varepsilon_{\mathrm{r}} / \partial H=2.1 \times 10^{-6}$.

If the relative permittivity of all the surroundings of the coil would be equal and would change equally with humidity, the capacitance would change according to $\partial C_{\mathrm{sens}} / \partial \varepsilon_{\mathrm{r}}=C_{\mathrm{sens}, 0} / \varepsilon_{\mathrm{r}, 0}=$ $\varepsilon_{\mathrm{r}, 0}^{-1} S R F_{\mathrm{sens}}^{-2} L_{\mathrm{sens}}^{-1}$, with $C_{\mathrm{sens}, 0}$ the nominal capacitance of the coil and $\varepsilon_{\mathrm{r}, 0}$ the nominal relative permittivity of the surroundings. However, for a coil attached to a probe, these conditions are not met. Typically, the $\varepsilon_{\mathrm{r}}$ of the probe material is different from the one of air gap and is ideally not influenced by humidity change. To account for these effects, a constant $k$ that depends on the geometry and materials of the probe is introduced, so that the equation becomes $\partial C_{\text {sens }} / \partial \varepsilon_{\mathrm{r}}=$ $k C_{\mathrm{sens}, 0} / \varepsilon_{\mathrm{r}, 0}=k \varepsilon_{\mathrm{r}, 0}^{-1} S R F_{\mathrm{sens}}^{-2} L_{\mathrm{sens}}^{-1}$, with $\varepsilon_{\mathrm{r}}$ the relative permittivity of the air gap.

As an example, a probe with the coil of Figure 2a was modelled. The probe is located at a 100 $\mu \mathrm{m}$ standoff from the target and is made of FR4 PCB material. At $360 \mu \mathrm{m}$ from the back of the coil a shield is placed. Figure $2 \mathrm{~b}$ shows the magnetic and electric field corresponding to the SRF of the coil, as calculated using a finite element model in Comsol. The coil has a lumped capacitance of $C_{\mathrm{sens}, 0}=$ $0.49 \mathrm{pF}$ and a sensitivity of $\partial C_{\text {sens }} / \partial \varepsilon_{\mathrm{r}}=0.17 \mathrm{pF}$, leading to $k=0.35$.

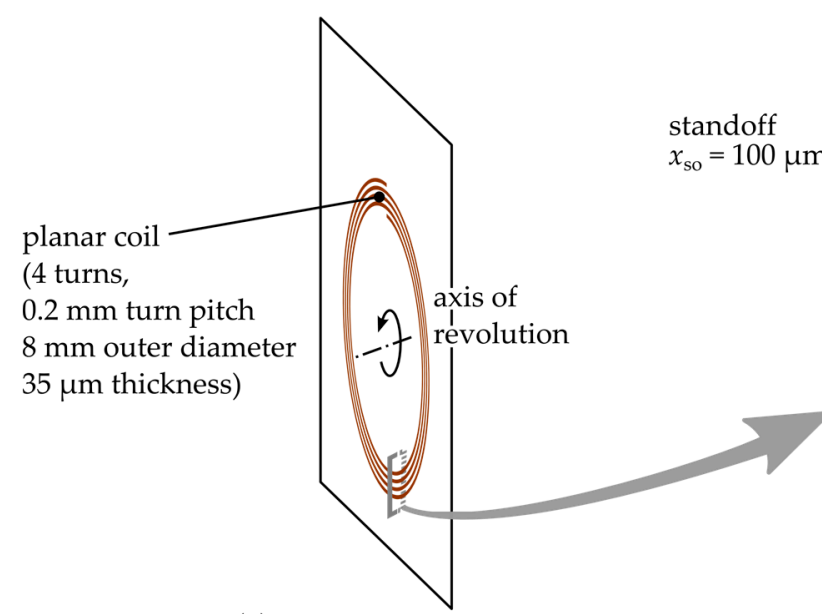

(a)

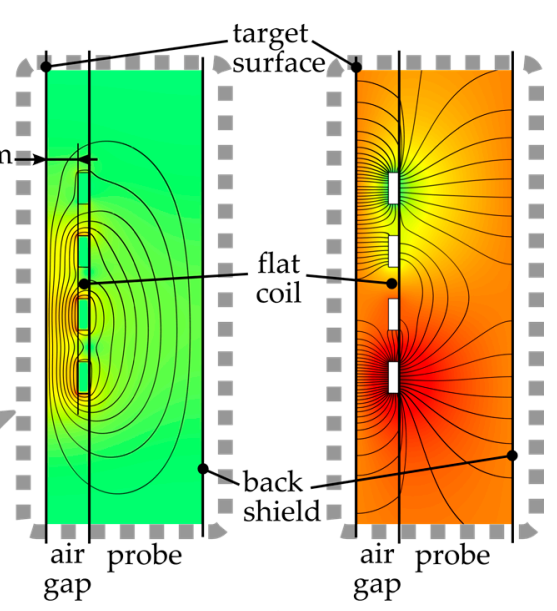

(b)

Figure 2. (a) The coil geometry used in this study. (b) Magnetic (left) and electric (right) field lines of the mode corresponding to the SRF of the coil.

The sensitivity of the capacitance to humidity can be expressed as

$$
\frac{\partial C_{\text {sens }}}{\partial H}=\frac{\partial C_{\text {sens }}}{\partial \varepsilon_{\mathrm{r}}} \frac{\partial \varepsilon_{\mathrm{r}}}{\partial H}=\frac{2.1 \times 10^{-6} k}{S R F_{\text {sens }}^{2} L_{\text {sens }}} .
$$

Combining Equations (4) and (7), the allowable humidity variation (in percent) as function of the ratio between the SRF and the excitation frequency is

$$
\Delta H=\left(\alpha^{2}-2+\frac{1}{\alpha^{2}}\right) \frac{S_{x}}{2.1 \times 10^{-6} k L_{\mathrm{sens}}} \Delta x_{\mathrm{app}} .
$$




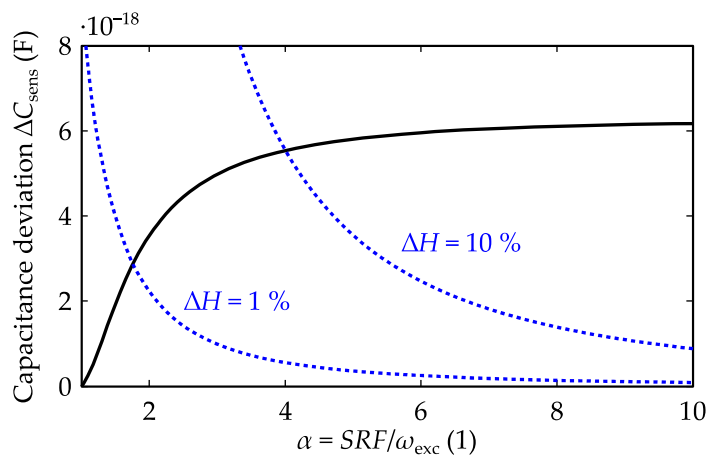

(a)

(a)

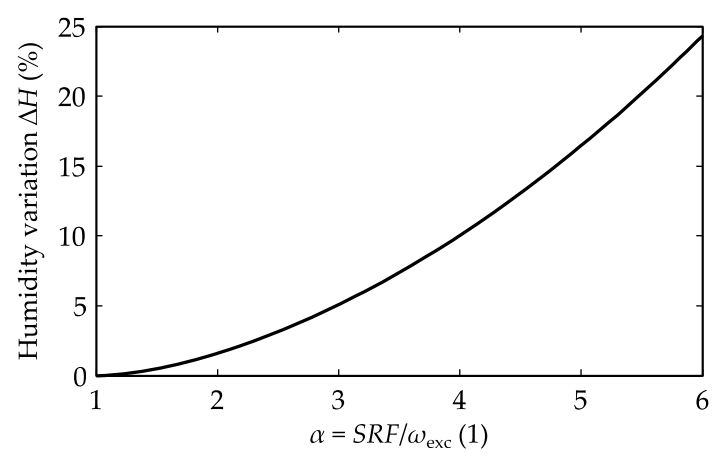

(b)

(b)

Figure 3. (a) Capacitance variation that leads to a $0.1 \mathrm{~nm}$ measurement error as a function of the normalized SRF (solid line) and the capacitance deviation resulting from a humidity variation of $1 \%$ and $10 \%$ (dashed lines); (b) Humidity variation as function of the normalized SRF.

Figure 3a shows the capacitance deviation that leads, for the selected coil geometry, to a $0.1 \mathrm{~nm}$ measurement error, as a function of the normalised SRF (solid line). Also the capacitance deviation resulting from a humidity deviation of $1 \%$ and $10 \%$ is shown (dashed lines). Figure $3 b$ provides the allowed humidity range as a function of the normalised SRF. For a relative humidity variation of $1 \%$ and $10 \%$, respectively, the SRF should be 1.8 and 4.0 times higher than the excitation frequency.

\section{Conclusions}

An important benefit of ECDSs over capacitive sensors is their insensitivity to environmental conditions. ECDSs that measure the reactance of the sensing coil, however, can not distinguish a change of the coil's parasitic capacitance from a change in the coil's inductance, leading to a certain sensitivity to environmental conditions.

The parasitic capacitance of the sensing coil can be characterised using the coil's SRF. An expression is derived for the humidity variation that leads to certain error on the displacement measurement. The expression depends, amongst others, on the ratio between the SRF and the excitation frequency. The knowledge of this relation allows to make a trade-off between the SRF and the humidity specifications of the sensor and can be used as input for the coil and probe design.

Acknowledgments: This research is funded by the Dutch Organisation for Scientific Research (NWO).

Conflicts of Interest: The authors declare no conflict of interest. The founding sponsors had no role in the design of the study; in the collection, analyses, or interpretation of data; in the writing of the manuscript, and in the decision to publish the results.

\section{References}

1. Fleming, A.J. A review of nanometer resolution position sensors: Operation and performance. Sens. Actuators A Phys. 2013, 190, 106-126.

2. Chaturvedi, V.; Nabavi, M.R.; Vogel, J.G.; Nihtianov, S. Demodulation techniques for self-oscillating eddycurrent displacement sensor interfaces: A review. IEEE Sens. J. 2017, 17, 2617-2624.

3. Chaturvedi, V.; Vogel, J.G.; Nihtianov, S. Suppression efficiency of the correlated noise and drift of self-oscillating pseudo-differential eddy current displacement sensor. Procedia Eng. 2016, 168, 946-949.

4. Chaturvedi, V.; Nabavi, M.R.; Vogel, J.G.; Makinwa, K.A.A.; Nihtianov, S. A $0.6 \mathrm{~nm}$ resolution $19.8 \mathrm{~mW}$ eddy-current displacement sensor interface with $126 \mathrm{MHz}$ excitation. In Proceedings of the International Solid-State Circuits Conference, San Francisco, CA, USA, 5-9 February 2017.

5. Vogel, J.G.; Nihtianov, S. Study of the self-resonance frequency of a flat coil for an eddy-current position sensor. In Proceedings of the IEEE Sensors Conference, Orlando, FL, USA, 30 October-3 November 2016.

6. Gäbler, S.; Heuer, H.; Heinrich, G. Measuring and imaging permittivity of insulators using high-frequency eddy-current devices. IEEE Trans. Instrum. Meas. 2015, 64, 2227-2238. 
7. Lea, N. Notes on the stability of LC oscillators. J. Inst. Electr. Eng. III Radio Commun. Eng. 1945, 92, $261-274$.

8. Lawrence, M.G. The relationship between relative humidity and the dewpoint temperature in moist air: A simple conversion and applications. Bull. Am. Meteorol. Soc. 2005, 86, 225-233.

(c) (1) BY

(C) 2017 by the authors. Licensee MDPI, Basel, Switzerland. This article is an open access article distributed under the terms and conditions of the Creative Commons Attribution (CC BY) license (http://creativecommons.org/licenses/by/4.0/) 